\title{
EFFICIENCY OF DIFFERENT TREATMENTS ON COCOON PRESERVATION OF THE SILKWORM Bombyx mori L. AND DIFFERENT SUBSTANCES AND PACKAGE MATERIALS \\ Ashour, Azza T. ${ }^{1}$ and Naglaa H. El-Sinary ${ }^{2}$ \\ 1 Department of Economic Entomology and Pesticides, Faculty of Agriculture, Cairo University, Giza, Egypt. \\ 2 Department of Natural Products, National Center for Radiation Research and Technology, Atomic Energy Authority, Cairo, Egypt.
}

\begin{abstract}
Cocoons of silkworm Bombyx mori L. were irradiated with four different doses of gamma radiation; $10,20,40$ and $60 \mathrm{krad}$ to minimize the damaged occurred during storage. Irradiated cocoons were packaged in different kinds of sac materials as traditional gunny, polypropylene, printed paper, thick paper, thin and thick cotton textiles treated with chamomile and propolis powder to overcome. Pest infestation preservation period was six months with one month as investigation interval. Packages of thick paper and thin textile treated materials gave the best results for preservation of cocoons irradiated with higher doses of gamma radiation gave the best results. as there were no resulted damaged cocoons through six months preservation period.

Infestation test was carried out by irradiating adults of Dermestes maculates with $10 \mathrm{krad}$ dose of gamma ray and transferred into the treated packages made of different kinds of sac material to investigate their ability for infesting silkworm cocoons. The lowest percentages of damaged cocoons were recorded when irradiated adults infested cocoons stored in thick paper, thin and thick cotton textiles treated packages. Generally the highest damaged cocoons percentages were scored when cocoons preserved in packages made of traditional gunny, polypropylene and printed paper.
\end{abstract}

\section{INTRODUCTION}

Silkworm, Bombyx mori L. cocoons are attacked during storage by many pests and microbial agents (Gouda and Deviah, 1985). In Egypt, the most important pests are the Dermestids beetles (Coleoptera; Dermestidae); Dermests maculates, $D$. leechi and other beetles; carpet beetles, Anthrenus crustaceus, A. pimpinella and A. glorious (El-Sinary and Ashour, 2000). Fumigation by phosphine leads to the pesticides problem residual contact and contamination (Rajendran and Hujira, 2005). Because of the enormous deleterious effect of pesticides and fumigants used for reducing damaged cocoons caused by insect pests and microbial agents, which represents great problem during cocoon storage, transportation and reeling (Rajendra and Abhay, 2007), different countries have examined alterations including plant extracts and vegetable oils for protecting cocoons during storage. Addition of propolis powder to protect stored materials is considered a good antimicrobial agents (El-Kemal and Yazgan, 2002 and El-Sinary, 2007).

Preservation of silkworm cocoons by gamma radiation is recently approaches to protect cocoons from insect attack and diseases (Kumar et al., 1990). Packaging and material of packages which provide desicated media 
avoid damage of stored products or cocoons during storage, marketing, transportation and thereafter exportation (Somying et al., 2002).

In Egypt, sericulture is an effective tool of rural development as it is generate income and employment to rural family labor and youth at farm level and there are many difficulties of silk production and transferring the traditional rearing to modern sericulture practices (Mahmoud, 2002). This study was designed to reduce the percentage of damaged cocoons by insects or microbes which affects the reeling performance and turn production to poor quality silk and the percentage of damaged cocoons should not exceed more than 5.0\% (Dandin et al., 2005). Different tools were examined by gamma irradiation, chamomile and propolis powder in this study to minimize the defective cocoons caused by insects and microbial agents, as well as using different material for cocoons packaging to reduce moisture during storage.

\section{MATERIALS AND METHODS}

\section{Cocoons preparation:}

Eggs of $B$. moril L. Chinese hybrids 9F7X were obtained from the Sericulture Department, Plant Protection Research Institute Agricultural Research Center. Hatched eggs. Rearing method was carried out according of Krishnaswami (1978). Cocoons were collected and dried by hot air.

\section{Gamma irradiation:}

Cocoons were irradiated with four doses; $10,20,40$ and $60 \mathrm{Krad}$ of gamma ray at the Gamma Cell Irradiation Unit $\left({ }^{60} \mathrm{C}\right)$ source located at the National Center for Radiation Research and Technology (NCRRT), Atomic Energy Authority, Cairo.

\section{Packages preparation:}

Different materials were selected as backages for cocoons preservation for protection and provide desiccated media. The examined materials were polypropylene, traditional, gunny, printed paper, thick paper, thin and thick cotton textiles. With thickness of $0.05,0.42,0.04,0.08,0.18$ and $0.35 \mathrm{um}$. Thickness were determined in the Solid State Department located in the NCRRT by the Micrometer Device.

\section{Packages treatments:}

Chamomile Matricarea chamonilla flowers were dried in current air and grinded in electric machine. Packages were dusted by chamomile powder as pest deterrent agent and $0.5 \mathrm{gm}$ of propolis powder as antimicrobial agent. Fifty irradiated cocoons were transferred into each package. Each treatment was triplicated beside the untreated treatment as a control.

\section{Damaged cocoons determination:}

Packages contain cocoons (irradiated with gamma rays or unirradiated) were stored under laboratory conditions for six months. Different kinds of packages (treated with chamomile + propolis or untreated one) were investigated every month to determine and record damaged cocoons percentage during storage. 
Infestation test:

Each kind of package containing 50 cocoons was infested by ten pairs of (gamma irradiated with 10 kard or un-irradiated) males and females of $D$. maculates beetles. Damaged cocoons percentage was observed and recorded along three months with intervals 15 days. Each treatment was triplicated.

\section{Statistical analysis:}

The data obtained from the present study were statistically analyzed, whenever, the calculated "F" Values were significant at 5\% level (Snedecor and Cochran, 1980).

\section{RESULTS AND DISCUSSION}

Table (1); a, b, c, d, e and f screend the combined effect of gamma irradiation and treatment with chamomile and propolis on cocoons of silkworm, $B$. mori during storage and preserved in different kinds of packages sacs. Data showed determination of damaged cocoons percentage by $D$. maculates, $A$. crustaceus, different storage unspecified insects attack during 180 days storage period through 30 days investigation intervals. Best results were registered when irradiated cocoons stored in treated (chamomile + propolis) packages made of thick cotton textile followed by thin cotton textile and thick paper as they recorded $0.00 \%$ damaged cocoons especially with higher doses of gamma irradiated cocoons gave the best results. The higher insect infestation was registered with packages made of traditional gunny, polypropylene and printed paper, respectively. These findings in agreement with Farag (1986), who found that preservation of gamma irradiated garlic bulbs in carton (thick paper) packages decreased the fungal diseases infections and the infestation by the garlic moth for a long time of storage as compared with bad results obtained when unirradiated bulbs stored in burlap packages (traditional gunny). The same findings were stated by Abdalla (2004) who discovered that complete infestation by Corcyra cephalonica was occurred after six months when rice stored in polypropylene packages while occurred after four months when jute was used.

It was clearly noticed that the higher the dose of gamma irradiation the lower percentage of damaged cocoons. This finding is agreed with ElSinary and Ashour (2000) who reported that when cocoons of silkworm $B$. mori irradiated with higher doses of gamma radiation could be preserved for a long period with higher protection from insects and diseases attack. The obtained data shows the highly significant differences between storage of cocoons (irradiated or unirradiated) in treated packages with chamomile and propolis and untreated one. Chamomile and propolis were drastically decreased the percentage of damaged cocoons by insect pests. These were in conformity with Kio and Don (2003) who recommended to expose surfaces of dried packages material which were prepared for $B$. mori storage or transportation with different vegetable oils which reduced the development of the progeny of $D$. maculates. 
Table (1):-a: Combined effect of gamma irradiation M. chamonilla and propolis and in different packaging materials on storage performance of $B$. mori L. cocoons

\begin{tabular}{|c|c|c|c|c|c|c|c|}
\hline \multicolumn{8}{|c|}{$\begin{array}{l}\text { Kind of Package } \\
\text { Traditional gunny }\end{array}$} \\
\hline \multicolumn{8}{|c|}{ Damaged cocoons percentage (\%) } \\
\hline Doses of gamma irradiation(krad) & \multirow[b]{2}{*}{ * } & UI+UT & UI+TP & $10+\mathrm{TP}$ & \multicolumn{3}{|c|}{ 20+TP|40+TP|60+TP } \\
\hline Infestation and infection kinds & & 3.33 & 0.00 & 0.00 & 0.00 & 0.00 & 0.00 \\
\hline Investigations intervals (days) & ** & 0.00 & 0.00 & 0.00 & 0.00 & 0.00 & 0.00 \\
\hline 30 & *** & 0.00 & 0.00 & 0.00 & 0.00 & 0.00 & 0.00 \\
\hline \multirow{3}{*}{60} & & 6.67 & 1.33 & 0.00 & 0.00 & 0.00 & 0.00 \\
\hline & ** & 0.00 & 0.00 & 0.00 & 0.00 & 0.00 & 0.00 \\
\hline & *** & 0.00 & 0.00 & 0.00 & 0.00 & 0.00 & 0.00 \\
\hline \multirow{3}{*}{90} & * & 10.00 & 4.00 & 0.00 & 0.00 & 0.00 & 0.00 \\
\hline & ** & 0.00 & 0.00 & 0.00 & 0.00 & 0.00 & 0.00 \\
\hline & *** & 0.00 & 0.00 & 0.00 & 0.00 & 0.00 & 0.00 \\
\hline \multirow{3}{*}{120} & * & 13.33 & 7.33 & 3.33 & 3.33 & 0.00 & 0.00 \\
\hline & ** & 5.33 & 3.33 & 0.00 & 0.00 & 0.00 & 0.00 \\
\hline & *** & 2.00 & 1.33 & 0.00 & 0.00 & 0.00 & 0.00 \\
\hline \multirow{3}{*}{150} & * & 18.00 & 10.00 & 6.00 & 5.33 & 3.33 & 1.33 \\
\hline & ** & 8.67 & 6.67 & 3.33 & 2.00 & 0.00 & 0.00 \\
\hline & 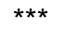 & 4.67 & 3.33 & 2.00 & 0.00 & 0.00 & 0.00 \\
\hline \multirow{3}{*}{180} & * & 20.67 & 12.67 & 10.00 & 6.33 & 4.67 & 3.33 \\
\hline & ** & 14.67 & 13.33 & 5.33 & 4.67 & 0.00 & 0.00 \\
\hline & *** & 9.33 & 6.33 & 7.33 & 3.33 & 0.00 & 0.00 \\
\hline L.S.D. & & & & & & & \\
\hline $\begin{array}{l}0.05 \\
0.01\end{array}$ & & $\begin{array}{l}3.9 \\
8.2\end{array}$ & $\begin{array}{l}2.9 \\
5.7\end{array}$ & $\begin{array}{l}3.1 \\
5.8\end{array}$ & $\begin{array}{l}2.3 \\
4.0\end{array}$ & $\begin{array}{l}1.9 \\
3.8\end{array}$ & $\begin{array}{l}1.0 \\
2.3\end{array}$ \\
\hline
\end{tabular}

Table (1):-b:

\begin{tabular}{|c|c|c|c|c|c|c|c|}
\hline \multicolumn{8}{|c|}{ Kind of Package } \\
\hline \multicolumn{8}{|c|}{ Damaged cocoons percentage (\%) } \\
\hline $\begin{array}{c}\text { Doses of gamma irradiation } \\
(\mathrm{krad})\end{array}$ & \multirow[b]{2}{*}{ * } & UI+UT & $\begin{array}{l}\mathrm{UI}+\mathrm{T} . \\
\mathrm{C}+\mathrm{P}\end{array}$ & \multicolumn{4}{|c|}{$10+$ T. $\mid 20+$ T. $\mid 40+$ T. $\mid 60+T}$. \\
\hline Infestation and infection kinds & & 1.33 & 0.00 & 0.00 & 0.00 & 0.00 & 0.00 \\
\hline Investigations intervals (days) & ** & 0.00 & 0.00 & 0.00 & 0.00 & 0.00 & 0.00 \\
\hline 30 & *** & 0.00 & 0.00 & 0.00 & 0.00 & 0.00 & 0.00 \\
\hline \multirow{3}{*}{60} & * & 5.33 & 0.00 & 0.00 & 0.00 & 0.00 & 0.00 \\
\hline & ** & 0.00 & 0.00 & 0.00 & 0.00 & 0.00 & 0.00 \\
\hline & *** & 0.00 & 0.00 & 0.00 & 0.00 & 0.00 & 0.00 \\
\hline \multirow{3}{*}{90} & * & 10.00 & 2.67 & 2.00 & 0.00 & 0.00 & 0.00 \\
\hline & ** & 1.33 & 0.00 & 0.00 & 0.00 & 0.00 & 0.00 \\
\hline & *** & 0.00 & 0.00 & 0.00 & 0.00 & 0.00 & 0.00 \\
\hline \multirow{3}{*}{120} & * & 11.33 & 4.67 & 3.33 & 1.33 & 0.00 & 0.00 \\
\hline & ** & 4.00 & 3.33 & 0.00 & 0.00 & 0.00 & 0.00 \\
\hline & $\star \star \star *$ & 0.00 & 1.33 & 0.00 & 0.00 & 0.00 & 0.00 \\
\hline \multirow{3}{*}{150} & * & 14.67 & 8.00 & 6.00 & 2.67 & 0.00 & 0.00 \\
\hline & ** & 5.33 & 5.33 & 0.00 & 0.00 & 0.00 & 0.00 \\
\hline & $\star \star * *$ & 3.33 & 2.00 & 0.00 & 0.00 & 0.00 & 0.00 \\
\hline \multirow{3}{*}{180} & $*$ & 19.33 & 10.67 & 8.00 & 4.00 & 3.33 & 2.67 \\
\hline & ** & 10.67 & 7.33 & 0.00 & 0.00 & 0.00 & 0.00 \\
\hline & $\star \star \star *$ & 7.33 & 5.33 & 0.00 & 0.00 & 0.00 & 0.00 \\
\hline $\begin{array}{c}\text { L.S.D. } \\
0.05 \\
0.01\end{array}$ & & $\begin{array}{l}3.5 \\
72\end{array}$ & $\begin{array}{l}2.8 \\
42\end{array}$ & $\begin{array}{l}1.9 \\
36\end{array}$ & $\begin{array}{l}2.0 \\
32\end{array}$ & $\begin{array}{l}1.1 \\
200\end{array}$ & $\begin{array}{l}1.1 \\
19\end{array}$ \\
\hline
\end{tabular}

UT = unirradiated cocoons, UT = untreated packages
TP = treated package with chamomile and propolis.

${ }^{*}=$ D. maculates, ${ }^{\star *}=$ carpet beetles, $\quad{ }^{* \star *}=$ different unspecified insects, 
J. Agric. Sci. Mansoura Univ., 33 (6), June, 2008

Table (1):-c:

\begin{tabular}{|c|c|c|c|c|c|c|c|}
\hline \multicolumn{8}{|c|}{$\begin{array}{c}\begin{array}{c}\text { Kind of Package } \\
\text { Printed paper }\end{array} \\
\end{array}$} \\
\hline \multicolumn{8}{|c|}{ Damaged cocoons percentage (\%) } \\
\hline $\begin{array}{c}\text { Doses of gamma irradiation } \\
(\mathrm{krad})\end{array}$ & \multirow[b]{2}{*}{ * } & UI+UT & $\begin{array}{c}\mathrm{Ul}+\mathrm{T} \\
\mathrm{C}+\mathrm{P}\end{array}$ & 10 & 20 & 40 & 60 \\
\hline Infestation and infection kinds & & 0.00 & 0.00 & 0.00 & 0.00 & 0.00 & 0.00 \\
\hline Investigations intervals (days) & ** & 0.00 & 0.00 & 0.00 & 0.00 & 0.00 & 0.00 \\
\hline 30 & *** & 0.00 & 0.00 & 0.00 & 0.00 & 0.00 & 0.00 \\
\hline \multirow{3}{*}{60} & * & 2.67 & 2.00 & 0.00 & 0.00 & 0.00 & 0.00 \\
\hline & ** & 0.00 & 0.00 & 0.00 & 0.00 & 0.00 & 0.00 \\
\hline & *** & 0.00 & 0.00 & 0.00 & 0.00 & 0.00 & 0.00 \\
\hline \multirow{3}{*}{90} & * & 6.33 & 4.67 & 1.33 & 0.00 & 0.00 & 0.00 \\
\hline & ** & 1.33 & 1.33 & 0.00 & 0.00 & 0.00 & 0.00 \\
\hline & *** & 0.00 & 0.00 & 0.00 & 0.00 & 0.00 & 0.00 \\
\hline \multirow{3}{*}{120} & ${ }^{*}$ & 9.33 & 6.33 & 2.00 & 0.00 & 0.00 & 0.00 \\
\hline & ** & 2.67 & 2.00 & 0.00 & 0.00 & 0.00 & 0.00 \\
\hline & 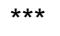 & 2.67 & 2.00 & 2.00 & 1.33 & 1.33 & 0.00 \\
\hline \multirow{3}{*}{150} & * & 12.67 & 9.33 & 4.67 & 0.00 & 0.00 & 0.00 \\
\hline & ** & 4.67 & 2.67 & 0.00 & 0.00 & 0.00 & 0.00 \\
\hline & *** & 3.33 & 2.67 & 2.67 & 2.00 & 1.33 & 0.00 \\
\hline \multirow{3}{*}{180} & ${ }^{*}$ & 14.67 & 11.33 & 6.33 & 2.67 & 1.33 & 0.00 \\
\hline & ** & 6.00 & 4.67 & 2.00 & 0.00 & 0.00 & 0.00 \\
\hline & $\star * *$ & 5.33 & 4.00 & 3.33 & 3.33 & 2.00 & 0.00 \\
\hline L.S.D. & & & & & & & \\
\hline $\begin{array}{l}0.05 \\
0.01\end{array}$ & & $\begin{array}{l}4.1 \\
8.3\end{array}$ & $\begin{array}{l}3.8 \\
6.1\end{array}$ & $\begin{array}{l}2.8 \\
4.0\end{array}$ & $\begin{array}{l}2.0 \\
3.4\end{array}$ & $\begin{array}{l}1.5 \\
2.5\end{array}$ & $\begin{array}{c}0.67 \\
1.2\end{array}$ \\
\hline
\end{tabular}

Table (1):-d:

\begin{tabular}{|c|c|c|c|c|c|c|c|}
\hline \multicolumn{8}{|c|}{$\begin{array}{c}\text { Kind of Package } \\
\text { Thick paper }\end{array}$} \\
\hline \multicolumn{8}{|c|}{ Damaged cocoons percentage (\%) } \\
\hline $\begin{array}{c}\text { Doses of gamma irradiation } \\
(\mathrm{krad})\end{array}$ & \multirow[b]{2}{*}{ * } & UI+UT & $\begin{array}{c}\mathrm{Ul}+\mathrm{T} \\
\mathrm{C}+\mathrm{P}\end{array}$ & 10 & 20 & 40 & 60 \\
\hline Infestation and infection kinds & & 0.00 & 0.00 & 0.00 & 0.00 & 0.00 & 0.00 \\
\hline Investigations intervals (days) & ** & 0.00 & 0.00 & 0.00 & 0.00 & 0.00 & 0.00 \\
\hline 30 & *** & 0.00 & 0.00 & 0.00 & 0.00 & 0.00 & 0.00 \\
\hline \multirow{3}{*}{60} & & 0.00 & 0.00 & 0.00 & 0.00 & 0.00 & 0.00 \\
\hline & ** & 0.00 & 0.00 & 0.00 & 0.00 & 0.00 & 0.00 \\
\hline & *** & 0.00 & 0.00 & 0.00 & 0.00 & 0.00 & 0.00 \\
\hline \multirow{3}{*}{90} & * & 2.67 & 0.00 & 0.00 & 0.00 & 0.00 & 0.00 \\
\hline & ** & 0.00 & 0.00 & 0.00 & 0.00 & 0.00 & 0.00 \\
\hline & *** & 0.00 & 0.00 & 0.00 & 0.00 & 0.00 & 0.00 \\
\hline \multirow{3}{*}{120} & & 3.33 & 0.00 & 0.00 & 0.00 & 0.00 & 0.00 \\
\hline & ** & 0.00 & 0.00 & 0.00 & 0.00 & 0.00 & 0.00 \\
\hline & $\star * *$ & 0.00 & 0.00 & 0.00 & 0.00 & 0.00 & 0.00 \\
\hline \multirow{3}{*}{150} & * & 5.33 & 2.67 & 0.00 & 0.00 & 0.00 & 0.00 \\
\hline & ** & 2.00 & 1.33 & 0.00 & 0.00 & 0.00 & 0.00 \\
\hline & $\star \star \star *$ & 2.00 & 0.00 & 0.00 & 0.00 & 0.00 & 0.00 \\
\hline \multirow{3}{*}{180} & & 8.00 & 4.67 & 2.00 & 0.00 & 0.00 & 0.00 \\
\hline & $\star *$ & 4.67 & 2.67 & 1.33 & 0.00 & 0.00 & 0.00 \\
\hline & $* * *$ & 2.00 & 0.00 & 0.00 & 0.00 & 0.00 & 0.00 \\
\hline $\begin{array}{c}\text { L.S.D. } \\
0.05 \\
0.01\end{array}$ & & $\begin{array}{l}2.2 \\
4.0\end{array}$ & $\begin{array}{l}0.94 \\
2.2\end{array}$ & $\begin{array}{l}0.4 \\
1.1\end{array}$ & $\begin{array}{l}- \\
-\end{array}$ & $\begin{array}{l}- \\
-\end{array}$ & $\begin{array}{l}- \\
-\end{array}$ \\
\hline
\end{tabular}

UI = unirradiated cocoons, UT = untreated packages,

TP = treated package with chamomile and propolis.

${ }^{*}=$ D. maculates, ${ }^{\star \star}=$ carpet beetles, ${ }^{\star \star \star}=$ different unspecified insects, 
Table (1):-e:

\begin{tabular}{|c|c|c|c|c|c|c|c|}
\hline & $\begin{array}{l}\text { Kind } \\
\text { hin }\end{array}$ & $\begin{array}{l}\text { of Pack } \\
\text { otton te }\end{array}$ & $\begin{array}{l}\text { ge } \\
\text { tile }\end{array}$ & & & & \\
\hline Damag & COC & ons pe & centag & (\%) & & & \\
\hline $\begin{array}{c}\text { Doses of gamma irradiation } \\
\text { (krad) }\end{array}$ & & UI+UT & $\begin{array}{l}\mathrm{UI}+\mathrm{T} . \\
\mathrm{C}+\mathrm{P}\end{array}$ & 10 & 20 & 40 & 60 \\
\hline Infestation and infection kinds & * & 0.00 & 0.00 & 0.00 & 0.00 & 0.00 & 0.00 \\
\hline Investigations intervals (days) & ** & 0.00 & 0.00 & 0.00 & 0.00 & 0.00 & 0.00 \\
\hline 30 & *** & 0.00 & 0.00 & 0.00 & 0.00 & 0.00 & 0.00 \\
\hline & * & 00.0 & 0.00 & 0.00 & 0.00 & 0.00 & 0.00 \\
\hline 60 & $* *$ & 0.00 & 0.00 & 0.00 & 0.00 & 0.00 & 0.00 \\
\hline & *** & 0.00 & 0.00 & 0.00 & 0.00 & 0.00 & 0.00 \\
\hline & * & 0.00 & 0.00 & 0.00 & 0.00 & 0.00 & 0.00 \\
\hline 90 & ** & 0.00 & 0.00 & 0.00 & 0.00 & 0.00 & 0.00 \\
\hline & *** & 0.00 & 0.00 & 0.00 & 0.00 & 0.00 & 0.00 \\
\hline & ${ }^{*}$ & 2.67 & 0.00 & 0.00 & 0.00 & 0.00 & 0.00 \\
\hline 120 & ** & 0.00 & 0.00 & 0.00 & 0.00 & 0.00 & 0.00 \\
\hline & *** & 0.00 & 0.00 & 0.00 & 0.00 & 0.00 & 0.00 \\
\hline & * & 4.67 & 1.33 & 0.00 & 0.00 & 0.00 & 0.00 \\
\hline 150 & ** & 1.33 & 0.00 & 0.00 & 0.00 & 0.00 & 0.00 \\
\hline & *** & 0.00 & 0.00 & 0.00 & 0.00 & 0.00 & 0.00 \\
\hline & * & 7.33 & 3.33 & 2.00 & 0.00 & 0.00 & 0.00 \\
\hline 180 & $\star *$ & 3.00 & 2.00 & 1.33 & 0.00 & 0.00 & 0.00 \\
\hline & *** & 1.33 & 0.00 & 0.00 & 0.00 & 0.00 & 0.00 \\
\hline $\begin{array}{c}\text { L.S.D. } \\
0.05 \\
0.01\end{array}$ & & $\begin{array}{l}1.3 \\
2.0\end{array}$ & $\begin{array}{l}0.4 \\
1.4\end{array}$ & $\begin{array}{l}0.4 \\
1.3\end{array}$ & - & - & - \\
\hline
\end{tabular}

Table (1):-f:

\begin{tabular}{|c|c|c|c|c|c|c|c|}
\hline \multicolumn{8}{|c|}{$\begin{array}{c}\text { Kind of Package } \\
\text { Thick cotton textile }\end{array}$} \\
\hline Damag & coc & \multirow{2}{*}{ Ons pe } & \multirow{2}{*}{$\begin{array}{c}\text { centag } \\
\text { Ul+ T. } \\
\text { C+P }\end{array}$} & (\%) & \multirow[b]{2}{*}{20} & \multirow[b]{2}{*}{40} & \multirow[b]{2}{*}{60} \\
\hline Doses of gamma irradiation & \multirow[b]{2}{*}{ * } & & & $\frac{10}{10}$ & & & \\
\hline Infestation and infection kinds & & 0.00 & 0.00 & 0.00 & 0.00 & 0.00 & 0.00 \\
\hline Investigations intervals (days) & ** & 0.00 & 0.00 & 0.00 & 0.00 & 0.00 & 0.00 \\
\hline 30 & *** & 0.00 & 0.00 & 0.00 & 0.00 & 0.00 & 0.00 \\
\hline \multirow{3}{*}{60} & & 0.00 & 0.00 & 0.00 & 0.00 & 0.00 & 0.00 \\
\hline & ** & 0.00 & 0.00 & 0.00 & 0.00 & 0.00 & 0.00 \\
\hline & *** & 0.00 & 0.00 & 0.00 & 0.00 & 0.00 & 0.00 \\
\hline \multirow{3}{*}{90} & * & 0.00 & 0.00 & 0.00 & 0.00 & 0.00 & 0.00 \\
\hline & ** & 0.00 & 0.00 & 0.00 & 0.00 & 0.00 & 0.00 \\
\hline & *** & 0.00 & 0.00 & 0.00 & 0.00 & 0.00 & 0.00 \\
\hline \multirow{3}{*}{120} & * & 0.00 & 0.00 & 0.00 & 0.00 & 0.00 & 0.00 \\
\hline & ** & 0.00 & 0.00 & 0.00 & 0.00 & 0.00 & 0.00 \\
\hline & *** & 0.00 & 0.00 & 0.00 & 0.00 & 0.00 & 0.00 \\
\hline \multirow{3}{*}{150} & * & 2.67 & 0.00 & 0.00 & 0.00 & 0.00 & 0.00 \\
\hline & $* *$ & 0.00 & 0.00 & 0.00 & 0.00 & 0.00 & 0.00 \\
\hline & $\star \star \star *$ & 0.00 & 0.00 & 0.00 & 0.00 & 0.00 & 0.00 \\
\hline \multirow{3}{*}{180} & * & 4.67 & 2.00 & 0.00 & 0.00 & 0.00 & 0.00 \\
\hline & $\star *$ & 2.00 & 1.33 & 0.00 & 0.00 & 0.00 & 0.00 \\
\hline & $\star \star \star *$ & 2.00 & 1.33 & 0.00 & 0.00 & 0.00 & 0.00 \\
\hline $\begin{array}{c}\text { L.S.D. } \\
0.05\end{array}$ & & 0.8 & 0.4 & - & - & - & - \\
\hline & & & & & & & \\
\hline
\end{tabular}

UI = unirradiated cocoons, UT = untreated packages,

TP = treated package with chamomile and propolis.

${ }^{*}=$ D. maculates, ${ }^{\star \star}=$ carpet beetles, $\quad{ }^{\star \star \star}=$ different unspecified insects, 
The deterrent effect of chamomile was also discussed by Rizk et al. (2001) who observed that chamomile was effective to reduce the damage of Phthorimaea operculella which cause great damage to potato tubers during storage. Recently Khadadad and Mehdi (2007) noticed that chamomile, M. chamonilla flowers extract has biological activity against Acari ixodidae. The antimicrobial agent, propolis was discussed by many authors; Brumfitt et al. (1990); El-Kemal and Yazgan (2002) and El-Sinary, (2007). They found that propolis considered a good antimicrobial agent and has antibiotic activity helps to minimize or inhibit many kinds of microbial growth.

Table (2) respresents damaged cocoons percentages results from infestation by irradiated or un-irradiated adults of $D$. maculates in treated different kinds of packages with chamomile and propolis or untreated ones. Infestation percentages were recorded along a period of three months with investigation intervals of fifteen days. The lowest percentage of damaged cocoons $(0.00)$ was obtained with treated packages with chamomile and propolis of thick paper, thin and thick cotton textile. These data were highly significantly compared with the same treatment of traditional gunny, polypropylene and printed paper packages which scored 16.0, 15.0 and $3.33 \%$, respectively. The highest percentages of damaged cocoons were determined when unirradiated adults of $D$. maculates infested untreated packages with chamomile and propolis made of traditional gunny, polypropylene and printed paper $(50.67,30.33$ and $24.0 \%)$ respectively. While packages made of thick paper, thin and thick cotton textile recorded $17.33,10.0$ and 7.33 , respectively. It is clearly noticed that gamma irradiation of adults played an important role in reducing the damaged cocoons percentage during storage. This conclusion is in agreement with results gathered by Nilufur and Heather (2003) who examined the susceptibility of different developmental stages $D$. maculates to different gamma irradiation doses and concluded that doses were effective to distinct attack to packaged products, the same was discovered by Shokoohian (2003).

It could be concluded from all the previous results that storage of silkworm, B. mori in thick paper or thin and thick cotton textile packages gave the best results to cocoons preservation during storage for a long period till reeling or transportation results were improved when packaged were treated with chamomile and propolis powder. Gamma irradiated cocoons could be stored for a long time than unirradiated one as irradiation helped in killing many stages of insect pests or microbial agents may defect cocoons. Modification in storage methods may improve cocoon production in Egypt to reach the level of advanced productive countries. 
Ashour, Azza T.and Naglaa H. El-Sinary

2

4486 


\section{REFERENCES}

Abdalla, R.S. (2004): Effect of gamma irradiation and some plant substances on certain stored product insects. M.Sc. Thesis, Department of plant Protection, Ain Shams University, Cairo, Egypt, pp. 88.

Brumitt, W., Hamilton-Miller, J.M.T. and Franklin, I. (1990): Antibiotic activity of natural products: 1- Propolis. Microbes, 62:19-22.

Dandin, S.B.; Srinivasababu, G. K.; Chandrakanth, K.S. and Vinod,B.M. (2005): New modified card board mountages for quality cocoon production. The $20^{\text {th }}$ congress of the international sericultural commission $15^{\text {th }}-18^{\text {th }}$ December, Bangalore, India, V.1, Section 2: Bombyx mori.

El-Kemal, B.G. and Yazgan, F. (2002): Effects of antimicrobial agents on the survival and development of larvae of Pimpla turionellae I. (Hymenoptera; Ichneumonidae) reared on an artificial diet. Turk. J. Zool. 26:11-119.

El-Sinary, N.H. and Ashour, Azza, T. (2000): Effect of gamma rays on cocoons preservation of the silkworm, Bombyx mori I. Bull. End. Soc. Egypt, Econ, Ser., 27, (73).

El-Sinary, N.H. (2007): Modified diet for mass rearing of the potato tubermoth, phthorimaea operculella (zeller), (Gelechiidae: lepidoptera). J. Egypt. Ger. Soc. Zool. Vol. (53e): Entomology, 171188.

Farag, S.A. (1986): Studies on some marketable characters of irradiated garlic during storage. Ph.D. Horticulture Department, Faculty of Agriculture, Ain Shams University, Cairo, Egypt. Pp:212.

Gouda, R.R. and Deviah, M.C. (1985): Beetle pests infesting stored silk cocoons. Journal of Agricultural Sciences, 19 (120-21).

Khodadad, P. K. and Mehdi, R.A. (2007): Biological activity of chamomile (matricaria chamonilla) flowers extract against the survival and egg laying of the cattle fever tick (Acari ixodidae) J. Zhejiang Univ. Sci. B., 8(9) : 693-696.

Kio, N. and Don, P. (2003): Insecticidal activity of some vegitable oils against Dermestes maculatus de geer (coleoptera : dermestidae) Journal of Stored Products Research, V (25), Issue 2, 81-86.

Krishnaswami S. (1978): New technology of silkworm rearing,Csr and Si, Bull, 2, Csrti, Mysore, India.

Kumar, P. ; Jayap Rakas, C.A.; Kishore, R. and Seng Upta, K. (1990): Effect of gamma radiation on the reproductive potential of Uzi Fly. INDIAN Journal of Sericulture, 29(2) 295-296.

Nilufur, K. and Heather, N.M. (2003): Susceptibility of Dermestes maculatusde geer (coleoptera : dermestidae) to gamma radiation in a nitrogen atmosphere. Journal of stored products research, Volume 26, I. 4, pages: $227-232$.

Rajendra, R. and Abhay, S. (2007): Encyclopaedia of agriculture, common wealth Pub., New Delhi, Vol. 10, p: 28-71. 
Rajendran, S. and Hujira, P. (2005): Insect infestation in stored animal products. Journal of Stored Products Research, Volume 41, issue 1, pages 1-30.

Rizk, S.A.; Haiba, I.M. and El-Sinary, N.H. (2001): Combined effect of gamma irradiation and ten plants on potato tubermoth, Phthorimaea operculella (zeller) larval mortality. Pakistan Journal of Biological Science V (4), Issue (10), 1228-1231.

Shokoohian, A. (2003): The effect of gamma radiation on different developmental stages of Dermestes maculatus de geer (Coleoptera: dermestidae). Journal of Stored Products Research, Volume 13, I. 2, pages: 88-90.

Snedecor, G.W. and Cochran, W.G. (1980): Statistical methods: $7^{\text {th }}$ ed. lowa State Univ. Press, Ames., lowa U.S.A., pp. 225-269.

Somying,C.; Siriporn, B. and Saridiporn, C. (2002): Sericulture in Thailand. $21^{\text {st }}-25^{\text {th }}$ september. Queen sirikit national convention center Bangkok, Thailand. Xix ${ }^{\text {th }}$ Congress of the International Sericultural Commission Proceedings.

كفاءة استخدام معاملات ومواد وعبوات مختلفة لحفظ شرانق دودة الحرير التوتية Bombyx mori L.

عزة توفيق عاشور1

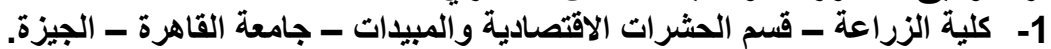
2- المركز القومي للبحوث وتكنولوجيا الإشعاع - هيئة الطاقة الذرية ـ مدينة نصر ـ القاهرة.

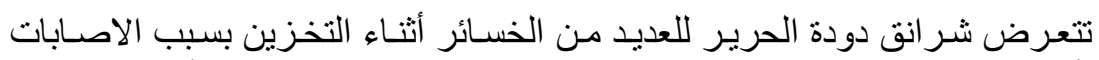

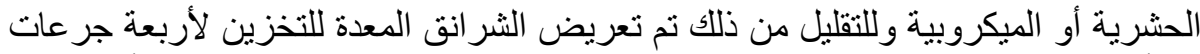

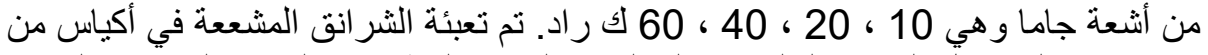

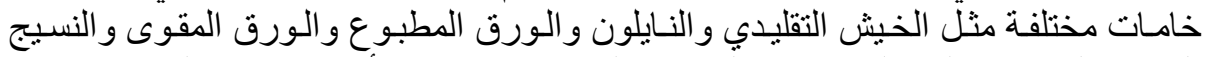

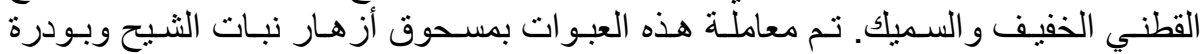

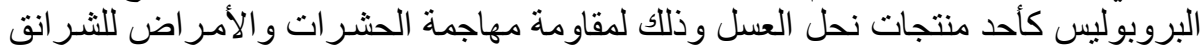

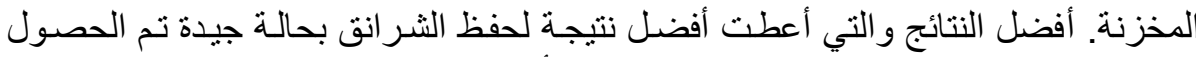

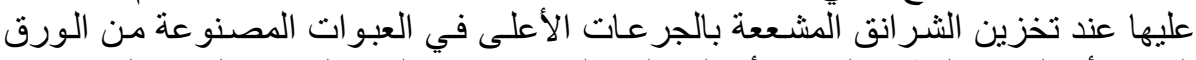

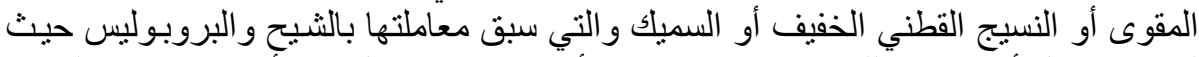

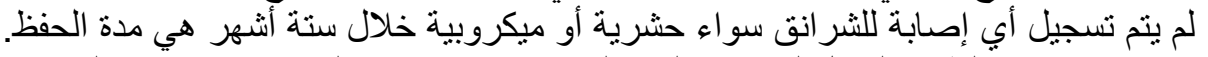

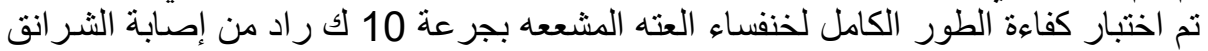

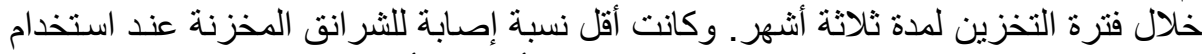

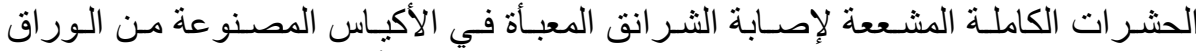

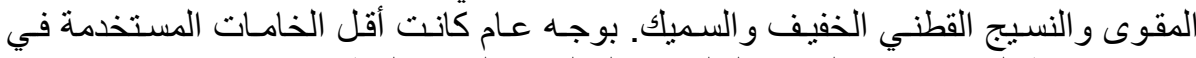
تخزين وحفظ الثر انت هي الخيش التقليدي والنايلون و الورق المطبوع. 


\section{J. Agric. Sci. Mansoura Univ., 33 (6): 4479 - 4488-, 2008}

Table (2): Damaged cocoons percentages infested by $D$. maculates in different kinds of packaging materials.

\begin{tabular}{|c|c|c|c|c|c|c|c|c|c|c|c|c|c|c|c|c|c|c|c|c|c|c|c|c|}
\hline \multirow{4}{*}{\begin{tabular}{|c|} 
Packages \\
$\begin{array}{c}\text { Infestation } \\
\text { by } D . \\
\text { maculatus } \\
\text { adults }\end{array}$ \\
\end{tabular}} & & & & & & & & & DaI & mag & ed c & $0 c 00$ & ons & (\%) & & & & & & & & & & \\
\hline & \multicolumn{4}{|c|}{ Traditional gunny } & \multicolumn{4}{|c|}{ Polypropylene } & \multicolumn{4}{|c|}{ Printed paper } & \multicolumn{4}{|c|}{ Thick paper } & \multicolumn{4}{|c|}{$\begin{array}{c}\text { Thin cotton } \\
\text { textile }\end{array}$} & \multicolumn{4}{|c|}{$\begin{array}{c}\text { Thick cotton } \\
\text { textile }\end{array}$} \\
\hline & \multicolumn{2}{|c|}{ TP } & \multicolumn{2}{|c|}{ UTP } & \multicolumn{2}{|c|}{ TP } & \multicolumn{2}{|c|}{ UTP } & \multicolumn{2}{|c|}{ TP } & \multicolumn{2}{|c|}{ UTP } & \multicolumn{2}{|r|}{ TP } & \multicolumn{2}{|c|}{ UTP } & \multicolumn{2}{|c|}{$\mathrm{TP}$} & \multicolumn{2}{|c|}{ UTP } & \multicolumn{2}{|c|}{ TP } & \multicolumn{2}{|c|}{ UTP } \\
\hline & I. & U.I & I. & U.I & $\mathrm{I}$. & U.I & I. & U.I & I. & U.I & I. & U.I & I. & U.I & I. & U.I & I. & U.I & I. & U.I & I. & U.I & I. & U.I \\
\hline \multicolumn{25}{|c|}{\begin{tabular}{|l|l|}
$\begin{array}{l}\text { Investigation } \\
\text { intervals } \\
\text { (days) }\end{array}$ \\
\end{tabular}} \\
\hline \begin{tabular}{|l|}
15 \\
15
\end{tabular} & \begin{tabular}{|l|}
0.0 \\
\end{tabular} & 0.0 & 0.0 & 4.0 & 0.0 & 0.0 & 0.0 & 3.33 & 0.0 & 0.0 & 0.0 & 0.0 & 0.0 & \begin{tabular}{l|l}
0 & 0.0
\end{tabular} & 0.0 & 0.0 & 0.0 & 0.0 & 0.0 & 0.0 & 0.10 & 0.0 & 0.0 & 0.0 \\
\hline 30 & 0.0 & 10.0 & 0.0 & 10.0 & 0.0 & 0.0 & 0.0 & 5.33 & 0.0 & 0.0 & 0.0 & 0.0 & 0.0 & \begin{tabular}{l|l}
0 & 0.0
\end{tabular} & 0.0 & 0.0 & 0.0 & 0.0 & 0.0 & 0.0 & 0.10 & 0.0 & 0.0 & 0.0 \\
\hline 45 & 0.0 & $|14.67|$ & 0.0 & 15.33 & 0.0 & 5.33 & 0.0 & 13.33 & 0.0 & 5.33 & 0.0 & 7.0 & 0.0 & \begin{tabular}{l|l}
0 & 0.0
\end{tabular} & 0.0 & 1.0 & 0.0 & 0.0 & 0.0 & 0.0 & 0.0 & 0.0 & 0.0 & 0.0 \\
\hline 60 & 0.0 & 20.0 & 10.67 & 22.0 & 0.0 & 10.67 & 0.0 & 20.0 & 0.0 & 8.67 & 5.33 & 10.6 & 870.0 & 05.33 & 0.0 & 3.33 & 0.0 & 1.33 & $\overline{0.0}(\mathrm{C}$ & 0.0 & 0.0 & 0.0 & 0.0 & 0.0 \\
\hline 75 & 7.33 & 30.67 & 15.33 & 341.33 & 10.0 & 14.67 & 12.67 & 25.33 & 2.67 & 12.0 & 10.67 & 718.6 & 370.0 & \begin{tabular}{l|l}
0 & 8.0
\end{tabular} & 6.0 & 9.33 & 0.0 & 2.67 & 1.335 & 5.33 & 0.02 & 2.67 & 0.02 & 2.67 \\
\hline 90 & 16.00 & 38.67 & 30.0 & 50.67 & 15.0 & 25.53 & 24.0 & 33.33 & 3.33 & 20.0 & 18.0 & 24.0 & 00.0 & 012.67 & 710.0 & 17.33 & 30.0 & 6.67 & & & 0.03 & & 3.33 & 7.33 \\
\hline \multicolumn{25}{|l|}{ IL.S.D. } \\
\hline 0.05 & 2.8 & & 3.2 & & 3.8 & & & & 2.9 & & & & 2.5 & & & & 2.2 & & & & 2.0 & & & \\
\hline 0.01 & 5.4 & & 7.1 & & 6.3 & & & & 7.0 & & & & 5.9 & & & & 4.1 & & & & 3.4 & & & \\
\hline
\end{tabular}

T.P = treated package withy chamomile and propolis, UTP = untreated package, I= irradiated cocoons and UI = unirradiated cocoons 